\section{ENCEPHALITIS LETHARGICA IN PREGNANCY *}

\author{
MARGARET SCHULZE, M.D. \\ SAN FRANCISCO
}

The case which I wish to report occurred in a pregnant woman of 35 . In reviewing the recent literature, I have found records of only seven other cases of encephalitis lethargica occurring in pregnant women. In the nona epidemic of 1890 , no cases complicating pregnancy are mentioned.

Though the fact is not always brought out in the smaller series, the disease appears to be far more conmon in the male sex than in the female, a point that was emphasized by Neal. ${ }^{1}$ Of 189 cases in recent English and American reports, only sixty-seven occurred in women and girls, and of these only thirtythree were in women of the child-bearing age. The mortality is, however, considerably higher in women than in men. Of 122 men, seventy-two recovered, twenty-three died, and in twenty-seven the outcome of the disease had not been determined at the time of the report; of the sixty-seven women, twenty-seven recovered, twenty-four died, and in sixteen the outcome was not yet known. The mortality rate in the pregnant women appears to be particularly high. Of the eight recorded cases, including my own, one recovered, five died, and in two the outcome was not reported.

The first case in this series was reported by Harris." This case occurred in the British epidemic in the spring of 1918, and was considered botulism, as were many of the earlier cases in England.

A young woman who was pregnant, almost at term, ate heartily of timned salmon, March 28. The following morning she developed diplopia, bilateral ptosis, marked drowsiness, and pyrexia up to $103 \mathrm{~F}$. She was delivered, April 2, without influence on the course of the disease, which had continued with double third nerve paralysis, drowsiness, pyrexia of $100 \mathrm{~F}$., retention of urine and increasingly active delirium up to the time of writing. Examination of the suspected food had shown a large gas-producing, anaerobic gram-positive, probably spore-bearing bacillus which had not as yet been identified.

Juncan " in May, 1918, reported a case of a married woman several months pregnant, who had been ill a few days. When first seen she was sitting by the fireside with both eyes closed. She was dull, but spoke when she was addressed. She could raise her eyelids, the right more than the left. There was herpes of the upper lip. The outcome of this case is not stated.

Neal ${ }^{1}$ has reported the only case with recovery at the time of writing.

This was a woman of 25 who was five months' pregnant. She had had an attack of influenza two weeks before the onset of the encephalitis, which began gradually with headache, chills and fever, vomiting, sweating and delirium. The spinal fluid showed great increase in cells and protein, a negative Wassermann test and negative guinea-pig inoculation for tuberculosis. Her condition remained the same for two weeks or more. Then she gradually recovered; the facial paralysis cleared up, and she had a normal delivery at term.

* From the Woman's Clinic, University of California Hospital.

* Owing to lack of space, this article is abbreviated in THE Journal by the omission of introductory notes on the history of the disease. The complete article appears in the author's reprints.

1. Neal, Josephine B.: Lethargic Encephalitis, Arch. Neurol. \& Psychiatry ₹: 271 (Sept. i) 1919.

2. Harris, W.: Lancet 1: 568 (April 20) 1918. McCaw, H. J.: Ibid. 1: 616 (April 27) 1918

3. Duncan, J. W.: Brit. M. J. 1:551 (May 11) 1918.
Sacts, ${ }^{4}$ in a series of seven cases, had three fatalities, of which two occurred in pregnant women. The duration of pregnancy is not stated in either case. In one there was a distinct history of influenza three months before the onset of the attack. Both of these cases presented all the ocular symptoms, the lethargy, and the ataxic and cerebellar symptoms. In one of the cases, in addition to the symptoms of poliencephalitis superior, there was also marked wasting of the muscles of the upper extremity and tremor of the hands. One case showed a spinal fluid with 20 cells. Acetone and diacetic acid were present in the urine in both cases. Both patients were extremely toxic. The question of inducing abortion was considered but rejecterl, and it was very questionable whether anything would have been gained by it. The total duration of the disease in each case was about four weeks.

Putnam's" case was in a woman of 26 , who had had two previous normal pregnancies and whose present pregnancy had been normal.

She was found lying on the floor apparently asleep. When aroused, she was weak and dizzy and took no notice of her sur roundings. She had no pain except a slight headache and no visual disturbances. Her face was expressionless and her eyes closed. The pulse was 114 and the temperature 99.4 F. The pupils were contracted and she tended to assume cataleptic postures. Her symptoms were at first thought merely hysterical, but her condition did not change, and the pulse rate and temperature remained elevated. Examination disclosed no paralyses, but some rigility of the extremities and a slight Kernig reflex with some pain on full extension. The eye muscles and face were normal. The leukocyte count was 14,000; the urine was negative; the Widal test proved negative; the spinal fluid showed 11 cells, gave a negative Noguchi reaction and reduced Haines' solution. For the first few days, she awoke spontaneously for food and defecation; later she could be aroused for these functions.

She was delivered three weeks after the onset of her illness. She did not arouse during the four hour labor. The child was stillborn; its heart had not been heard the whole day previous. Four days after delivery the patient died.

Bassoe ${ }^{6}$ reports a fatal case with postmortem examination in a woman of 34 , an octipara in the sixth month of pregnancy.

This-patient complained of listlessness, fatigue and headache. She had some nausea but no vomiting, and moderate fever. The facial expression was dull, and there was a slight facial palsy on the right side. The protrusion of the tongue was weak. The back was somewhat rigid, the deep reflexes on the left side were increased, but there was no rigidity of the extremities. The pupils and eyegrounds were normal. She was mentally dull, slightly confused and disoriented for time. Five weeks after the onset of the disease, hyperpyrexia and pulmonary edema developed and the patient died.

Postmortem examination revealed marked congestion of the veins of the pia, with edema and congestion of the brain and minute hemorrhages visible on the cut surfaces, especially in the white matter of the centrum ovale and the basal ganglions. The ventricles and their ependyma were of normal appearance. Sections from the frontal lobe showed marked distention of the vessels, especially the veins, but there were only very smiall lymphocytic infiltrations around some of the smaller vessels. In the left motor cortex, the pia was loose in texture with slight increase of cells. There was no definite sign of inflammation in the cortex itself. The left optic thalamus showed very extensive inflammation with large collections of mononuclear cells about the distended vessels. There were no large hemorrhages. All the sections

\footnotetext{
4. Sachs, B.: New York M. J. 109: 894 (May 24) 1919.

. Putnam, O.: J. Missouri M. A. 16:260 (August) 1919 971 (April 5) 1919. Epidemic Encephalitis (Nona), J. A. M. A. 72
} 
of the pons showed marked inflammatory changes, most pronounced in the dorsal portion, with numerous small hemorrhages. The bulb showed no inflammatory changes.

\section{REPORT OF AUTHOR'S CASE}

The case which $I$ wish to report is the following:

Mrs. N. A., a pregnant woman aged 35, entered the obstetric service of the University of California Hospital, April 23, 1919, complaining of edema of the legs and hands of one month's duration, and aching pains in both arms during the previous week. The pregnancy was one month past term. She had had one full term pregnancy six years before, com. plicated with marked edema, and some eye symptoms and pains in the legs during the last month, which had disappeared shortly after delivery. Her past history was otherwise negative. She had not had influenza, nor had any one in her immediate family.

Physical examination was negative except for the presence of a full term fetus in the left occipito-anterior position, varicosities of both legs with more edema of the legs than these could account for, and moderate edema of the hands.

Her mentality was rather low, but her mind seemed clear. Sensory examination disclosed a slight hyperesthesia to touch and pain over the posterior part of each shoulder; elsewhere sensation was normal. The pupillary reactions were normal. The deep reflexes were somewhat exaggerated. with a false patellar clonus. The left abdominal reflex was increased, the right was doubtful. There was a right sided Babinski reflex. Both hands showed a constant tremor. which increased if the patient tried to control it, but decreased if her attention was diverted. The blood pressure was 140 systolic and 95 diastolic. The urine showed the faintest possible trace of albumin with no casts. Both blood and urinary nitrogen were normal, and blood and urine cultures sterile. As all her teeth had been extracted dental roentgenograms were not taken and there were no other foci of infection demonstrable. The hemoglobin was 55 per cent. Sahli; the blood Wassermann reaction was negative. The temperature on the day of entry was $37.5 \mathrm{C}$. (99.5 F.), on the following day $37.2 \mathrm{C}$. $(98.9 \mathrm{~F}$.). After this it was normal for five days, until delivery. The pains in her arms persisted and at times were so severe as to require morphin for sleep. April 30, after five hours of normal labor, she was delivered of a child weighing $4,600 \mathrm{gm}$. (10 pounds). Following delivery she had a low grade fever, never higher than 37.6 ( $99.7 \mathrm{~F}$.) and therefore not requiring a uterine culture. On the tenth day the temperature reached $38 \mathrm{C}$. $(100.4 \mathrm{~F}$.). It was now noticed that she had become very dull mentally and seemed in a sort of stupor most of the time. She was easily aroused, and when addressed answered rationally and was clearly oriented. She complained of diplopia, and said that the pain in her arms kept her awake at night. A slight ptosis of the left lid and a right sided facial paralysis developed. The Babinski reflex had now disappeared, the other reflexes and the sensory findings remaining about the same. There were no signs of meningeal involvement.

A spinal puncture, May 15, yielded a clear fluid under slightly increased pressure. The Nonne and Noguchi reactions were negative, the cell count was twenty-six and Fehling's solution was reduced. The Wassermann reaction was negative and cultures were sterile. There was no web. A second puncture, May 15, was again sterile. There was a moderate leukocytosis, varying from 12,000 to 18,000 with 80 per cent. polymorphonuclears. A nasopharyngeal culture showed Bacillus infuenzae. The neurologic signs varied. She developed weakness of the muscles of mastication, tremor of the tongue with protrusion to the right, and then a right external rectus palsy.

About May 23, she began to improve markedly. Her mentality was much clearer, the tremor decreased, the tongue could be protruded normally, and the external rectus palsy was much less marked. The right arm reflexes were still increased, but the other reflexes were normal except for an atypical Oppenheim sign on the right side: The temperature became normal.
May 26, after getting up and walking about her bed without permission, she suddenly developed symptoms of pulmonary embolism and died in twenty minutes, twenty-six days after delivery.

Postmortem examination revealed thrombosis of both femoral veins and extensive bilateral pulmonary emboli, which occluded almost the entire pulmonary circulation. Macroscopically the brain was practically normal except for a few punctate hemorrhages on cut section in the region of the midbrain. The cerebrospinal fluid was normal in appearance and amount. Microscopic examination revealed the characteristic changes of lethargic encephalitis. There was congestion and slight round cell infiltration of the leptomeninges. There were marked perivascular infiltration and a few small hemorrhages about the vessels, cspecially of the midbrain and pons. There was no degeneration of the nerve cells. The medulla showed much less marked changes; the cerebral cortex was normal.

\section{Clinical Notes, Suggestions, and New Instruments}

\author{
ADMINISTRATION OF DIGITALIS BY "EgGLESTON \\ METHOD" \\ C.try Eggleston, M.D., New York
}

Many requests have been received for information as to the practical application of this method of administering digitalis, and therefore it seems advisable to publish a brief statement regarding the details involved. The reader is referred to the original paper ${ }^{2}$ for the data on which the method is based, and to the papers by S. Marx White and R. Edwin Morris, ${ }^{2}$ and by R. Edwin Morris," for comments on the method.

This method of digitalis dosage and administration is designed especially for rapid digitalization by oral administration. It depends on the establishment of an average total amount of digitalis which is required to produce full digitalization, or the minor toxic actions of digitalis. This total amount is expressed in terms of the activity of the drug and the patient's body weight in pounds. The activity of the drug is determined by the cat method of Hatcher, ${ }^{*}$ descriled in the Amcrican Journal of Pharmacy, the unit being the weight of dry drug, in milligrams, which is required to kill $1 \mathrm{~kg}$. of cat when a solution is injected slowly and continuously intravenously. This amount is called a cat unit. High grade specimens of digitalis, when not assayed by the cat method, may be regarded as having an average activity of $100 \mathrm{mg}$. to the cat unit. The average total amount of digitalis required for oral administration to man is 0.15 of one cat unit per pound of body weight.

$$
\text { CALCUlation OF AVERAge total AMOUNT }
$$

The calculation of the average total amount required by any given patient may be as follows:

1. The patient's weight is determined in pounds.

2. The cat unit of the digitalis is determined.

3. One of the following formulas is applied:

Formula I: $\frac{C \cdot U \cdot \times 0.15 \times \mathbb{W}}{1,000}=$ Grams of powdered leaf in total
amount
Formula II: $\frac{C \cdot U \cdot \times 0.15 \times W}{100}=$ Cuhic centimctcrs of tincture in
total amount
Formula III: $\frac{C \cdot U \cdot}{100} \times W=$ Cubic centimeters of infusion in total
amount

In these formulas, $C . C$. is the number of milligrams in one cat unit, and $W$ is the patient's body weight in pounds.

1. Eggleston, Cary: Digitalis Dosage, Arch. Int. Med. 16: I (July) 2. White, S. M., and Morris, R. E.: The Eggleston Method of Administering Digitalis, Arch. Int. Med. 21: 740 (June) 1918.

3. Morris, R. E.: Clinical Pharmacology of Digitalis, Minnesota Med. 1: 125 (Apri1) 1918

4. Hatcher, R. A., and Brody, J. G.: The Biological Standardization of Drugs, Am. J. Pharm. 82:360, 1910. 